\title{
The Effect of Particle Size on the Physical Characteristics and Drug-release Behavior of Mini-tablets
}

\author{
Awis Sukarni Mohmad Sabere ${ }^{1, *}$ and Mohd Muzamir Mahat ${ }^{2}$ \\ ${ }^{I}$ Kulliyyah of Pharmacy, International Islamic University Malaysia, Bandar Indera Mahkota, \\ 25200 Kuantan, Pahang, Malaysia \\ ${ }^{2}$ Textile Research Group, Faculty of Applied Sciences, Universiti Teknologi MARA, 40450 Shah Alam, \\ Selangor, Malaysia
}

('Corresponding author's e-mail: awissabere@iium.edu.my)

Received: 23 June 2020, Revised: 8 March 2021, Accepted: 23 March 2021

\begin{abstract}
This study aimed to investigate the influence of particle size of the excipients on the behavior of mini-tablets in terms of physical characteristics and drug release profiles. Material and methods: All of the mini-tablets based on HPMC, PEG 6K and PEO 8M PVP produced high quality mini-tablets from both sieve fractions while $90 \mathrm{~F}$ was very fragile. Results: Crushing strength values were the most pronounced difference between the mini-tablets from all formulations. The PEO 8M-based mini-tablets showed the highest values and deformation behavior instead of fracture. Water absorption and drug release profiles also showed intriguing and different results between the various formulations. Conclusion: The results suggested that even though different particle size has limited effect on the physical characteristic of the product, it may have a significant effect on its drug dissolution profile.
\end{abstract}

Keywords: Drug release profile, Mini-tablets, Particle size, Physical characterization

\section{Introduction}

Conventional tablets usually are round in shape with a minimum diameter of $7 \mathrm{~mm}$. On the other hand, mini-tablets are smaller, with a diameter between 2 and $3 \mathrm{~mm}$ or less [1]. Mini-tablets can either be filled into hard-shell capsules or compacted into larger tablets for controlled release formulation [2]. The process of manufacturing mini-tablets is similar to those of regular-sized tablets. However, mini-tablets manufacturing requires special attention in certain areas, such as the particle size distribution of the powders or granules [3].

The particle size of the powders or granules needs to be controlled during the manufacturing process. If the particle size is too large, only several particles can be fitted in the die. However, if the particles size is too fine, there is a possibility for the powder to pass through the gap between the die and the punch. Both scenarios will likely affect the content uniformity of the excipients and active ingredients, affecting the weight and active ingredients dosage of the mini-tablets. Since smaller particle size has larger total surface area, alteration of the particle size distribution may also affect the drug release profile of the mini-tablets.

This study evaluated the effect of particle size on the physical characteristics and drug-release behavior of the mini-tablets. Chloramphenicol, a broad-spectrum antibiotic for bacterial infection treatment, was used as the model drug. The formulations used in this study were prepared using direct compression to ensure that the investigation was primarily focused on particle size distribution only.

\section{Materials and methods}

\section{Materials}

Hydroxypropyl methylcellulose K4M (HPMC) was purchased from The DOW Chemical Company, USA. Polyethylene glycol 6000 (PEG 6K) was purchased from Clariant GmbH, Germany. Polyvinylpyrrolidone 90F (Kollidon 90F ${ }^{\mathrm{TM}}$ ) (PVP 90F) was provided by BASF The Chemical Company, Germany. Polyethylene oxide 8,000,000 (PEO 8M), chloramphenicol, calcium chloride, and sodium bicarbonate were obtained from Sigma Aldrich, Germany. Sodium stearyl fumarate (SSF) was obtained 
from Tokyo Chemical Industry, Japan. Sodium chloride was obtained from Fluka Chemie GmbH, Germany.

\section{Methods}

\section{Manufacturing process of the mini-tablets}

All the mini-tablets were prepared with $5 \% \mathrm{w} / \mathrm{w}$ chloramphenicol, $2 \% \mathrm{w} / \mathrm{w}$ SSF and $93 \% \mathrm{w} / \mathrm{w}$ polymer. The polymers employed in this study were HPMC, PEG 6K, PEO 8M and PVP 90F. Two sieve fractions were evaluated for each polymer, which were 125 to 180 and 180 to $250 \mu \mathrm{m}$. The drug and polymer were mixed using a Turbula mixer for $10 \mathrm{~min}$. SSF was added and mixed for another $5 \mathrm{~min}$. Mini-tablets were compressed with a rotary tablet press (Riva, Argentina) equipped with 2 mm normal concave plain punches at a compression force of $1.8 \pm 0.2 \mathrm{kN}$ [4]. Only 1 set of tooling was used during the process. Approximately, 100 mini-tablets per batch was manufactured for each formulation.

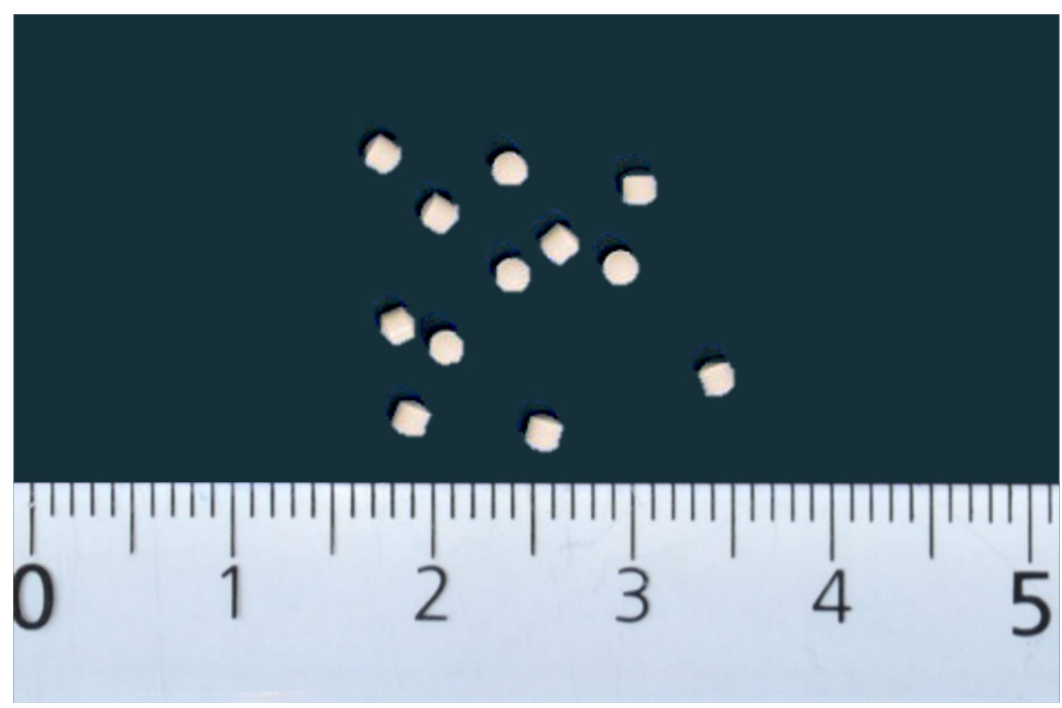

Figure 1 Manufactured mini-tablets.

\section{Physical characterization of the mini-tablets}

Weight uniformity

The weight uniformity of the mini-tablets was evaluated following the British Pharmacopoeia [5] method. Twenty tablets were selected randomly from each formulation and weighed individually. The mean weight and standard deviation were calculated.

\section{Measurement of mini-tablet dimensions}

The thickness of 10 random tablets from each formulation was measured with a digital caliper (Mitotoyo, Japan). The diameter was assumed to be constant at $2 \mathrm{~mm}$, based on the diameter of the punches.

\section{Friability}

The tablets' friability was determined by weighing 10 tablets as a unit, followed by placing the tablets in a friability tester drum (Copley Scientific, UK). The drum was configured to rotate for 100 revolutions at the speed of $25 \mathrm{rpm}$. The tablets were dedusted and re-weighted to calculate the percentage of friability using following equation:

$\mathrm{F}(\%)=\left(\frac{\mathrm{P}-\mathrm{P}^{\prime}}{\mathrm{P}}\right) \times 100$

where $\mathrm{P}$ is the initial weight of the tablets, and $\mathrm{P}$ ' is the final weight of the tablets. 
Crushing strength

Ten random tablets were assessed with a TA.TX2 texture analyer (Stable Microsystems Ltd, UK) according to the method by El-Gawad [6]. The mini-tablets placed on the fixed platform were crushed with an $11 \mathrm{~mm}$ diameter Perspex cylinder probe that was attached to the texture analyzer. This study's instrument parameters were: $1 \mathrm{~mm} / \mathrm{s}$ pre-test speed, $0.1 \mathrm{~mm} / \mathrm{s}$ test speed, $1 \mathrm{~mm} / \mathrm{s}$ post-test speed, $1 \mathrm{~mm}$ compression distance and $0.05 \mathrm{~N}$ trigger force. The force required to crush the tablets were recorded.

\section{Moisture uptake}

The mini-tablets' moisture uptake profile was monitored with dynamic vapor sorption (DVS) (Surface Measurement Systems, UK) at a fixed humidity level of $95 \pm 1 \% \mathrm{RH}$ for over $24 \mathrm{~h}$. Three random tablets were tested for each formulation.

\section{Drug release studies}

A modified flow-through dissolution apparatus with a $200 \mu \mathrm{L}$ chamber was used to assess the minitablets' drug release profile. Simulated tear fluid (STF) with a $\mathrm{pH}$ of 7.4 was prepared with $0.2 \% \mathrm{w} / \mathrm{w}$ sodium bicarbonate, $0.008 \% \mathrm{w} / \mathrm{w}$ calcium chloride and $0.67 \% \mathrm{w} / \mathrm{w}$ sodium chloride [7] and used as dissolution medium. The flow rate of the dissolution medium was fixed at $50 \mu \mathrm{L} / \mathrm{min}$. The samples were collected hourly for $9 \mathrm{~h}$. Drug concentration in the samples was quantified spectrophotometrically at 278 $\mathrm{nm}$. Three replicates were performed for each formulation.

\section{Statistical analysis}

The data were analyzed with the Statistical Package for Social Sciences (SPSS) and Microsoft Excel. Analysis of variance (ANOVA) was applied to determine any significant $(p<0.05)$ differences in the mini-tablets' physical parameters.

\section{Results and discussion}

Physical characterization of the mini-tablets

Table 1 summarizes the physical characterization data for the mini-tablets, except for vapor sorption. Each of the characterization studies is discussed in detail in the following sections.

Table 1 Summary of the physical characterization of mini-tablets (mean $\pm \mathrm{SD}$ ).

\begin{tabular}{cccccc}
\hline Polymer & $\begin{array}{c}\text { Sieve Fraction } \\
(\boldsymbol{\mu m})\end{array}$ & $\begin{array}{c}\text { Weight }(\mathbf{m g}) \\
(\mathbf{n}=\mathbf{2 0})\end{array}$ & $\begin{array}{c}\text { Thickness }(\mathbf{m m}) \\
(\mathbf{n}=\mathbf{1 0})\end{array}$ & $\begin{array}{c}\text { Friability }(\%) \\
(\mathbf{n}=\mathbf{3})\end{array}$ & $\begin{array}{c}\text { Crushing strength }(\mathbf{N}) \\
(\mathbf{n}=\mathbf{1 0})\end{array}$ \\
\hline HPMC & $125-180$ & $6.26 \pm 0.34$ & $1.89 \pm 0.03$ & $0.22 \pm 0.01$ & $12.43 \pm 1.74$ \\
HPMC & $180-250$ & $6.20 \pm 0.21$ & $1.91 \pm 0.04$ & $0.16 \pm 0.06$ & $17.11 \pm 2.55$ \\
PEG 6K & $125-180$ & $5.50 \pm 0.35$ & $1.58 \pm 0.04$ & $0.48 \pm 0.04$ & $8.24 \pm 1.29$ \\
PEG 6K & $180-250$ & $9.75 \pm 0.29$ & $2.79 \pm 0.04$ & $0.34 \pm 0.07$ & $12.10 \pm 1.38$ \\
PEO 8M & $125-180$ & $6.97 \pm 0.08$ & $2.22 \pm 0.01$ & $0.03 \pm 0.01$ & $43.18 \pm 1.44$ \\
PEO 8M & $180-250$ & $7.40 \pm 0.09$ & $2.33 \pm 0.02$ & $0.05 \pm 0.02$ & $40.07 \pm 2.06$ \\
PVP 90F & $125-180$ & $7.84 \pm 0.07$ & $2.43 \pm 0.01$ & $1.44 \pm 0.25$ & $5.84 \pm 0.59$ \\
PVP 90F & $180-250$ & $7.43 \pm 0.10$ & $2.34 \pm 0.02$ & $9.92 \pm 0.69$ & $5.64 \pm 0.99$ \\
\hline
\end{tabular}

\section{Weight uniformity}

The weight of all the mini-tablets was within the specification of British Pharmacopoeia [5]. Each polymer displayed a similar weight between the sieve fraction except for PEG $6 \mathrm{~K}$, where the larger particle size powder showed higher weight.

\section{Thickness}

The range of measured thickness values was between $1.89 \pm 0.03$ and $2.79 \pm 0.04 \mathrm{~mm}$. All batches displayed an acceptable level of variation, which was supported by the relative standard deviation (RSD) values. It could be suggested that all the mini-tablets from the same batch had a similar thickness. A statistically significant difference $(p<0.05)$ was observed between the 2 sieve fractions of PEO 8M and PVP 90F mini-tablets. PEG 6K mini-tablets showed a significant difference where the larger sieve fraction had thicker tablets than the smaller sieve fraction. There was no significant difference between the thickness of the 2 sieve fractions of HPMC mini-tablets. 


\section{Crushing strength}

The crushing strength results displayed a wide range of values between $5.64 \pm 0.99$ and $43.18 \pm$ $1.44 \mathrm{~N}$, as shown in Table 1. PVP 90F mini-tablets had the lowest crushing strength, followed by PEG $6 \mathrm{~K}, \mathrm{HPMC}$ and PEO 8M mini-tablets for both sieve fractions. There is a lack of crushing strength specification in the British Pharmacopoeia. However, based on previous studies on mini-tablets, the common value of hardness is in the range of 0.67 to $18.64 \mathrm{~N}$ [8,9]. Crushing strength value showed a correlation with the disintegration and dissolution profiles of the tablets, where higher crushing strength have longer disintegration and dissolution time [10].

PEO 8M mini-tablets from both sieve fractions had the highest crushing strength. These tablets displayed distinct behavior than the rest of the mini-tablets. Instead of breaking, the PEO 8M mini-tablets were just deformed. The recorded endpoint was the force to compress the tablets at the end of the probe's fixed-distance travel. Hence, the PEO mini-tablets measurements should not be over-interpreted even though they were consistent with low RSD values.

The rest of the mini-tablets exhibited typical breaking behavior. No significant difference $(p>0.05)$ was observed in the crushing strength of both PVP 90F mini-tablets sieve fractions. However, HPMC and PEG 6K mini-tablets showed significant differences $(p<0.01)$, with the larger sieve displaying greater crushing strength. This result is counter-intuitive since theoretically, smaller particle size fraction would have increased binding and greater resistance to crushing due to increased contact between the particles. These batches had higher RSD values, which were beyond $10 \%$. Even though a standard is absent in the British Pharmacopoeia, the crushing strength should be kept consistent since it may affect the tablets' dissolution behavior.

\section{Friability}

According to the British Pharmacopoeia [5], the percentage of weight loss should not exceed $1.0 \%$. All the mini-tablets, except for PVP 90F mini-tablets for both sieve fractions, passed the friability test. The losses in percentage of smaller and larger sieve fractions of PVP 90F were $1.44 \pm 0.25$ and $9.92 \pm$ $0.69 \%$, respectively.

These results were expected since, theoretically, higher crushing strength comes with lower friability. Friability test is essential since it mimics the external forces that the tablets will experience during the handling and transporting processes of the product from the manufacturing process to administration by the patient. The drug dosage can be reduced proportionally with the weight of the tablet. Therefore, the tablet's therapeutic outcome may be compromised with its weight loss [11].

\section{Moisture uptake}

Figure 1 shows the moisture uptake of the mini-tablets over $24 \mathrm{~h}$. PEO $8 \mathrm{M}(125-150 \mu \mathrm{m})$ minitablets showed the most significant changes in their weight after $24 \mathrm{~h}$, while the lowest weight gain was for PVP 90F $(180-250 \mu \mathrm{m})$ mini-tablets, at approximately 130 and $40 \%$ weight increment from the initial weight, respectively. PEG 6K and PEO 8M mini-tablets for both sieve fractions were completely dissolved at the end of the study. HPMC and PVP 90F mini-tablets for both sieve fractions absorbed water and showed swelling symptoms. 


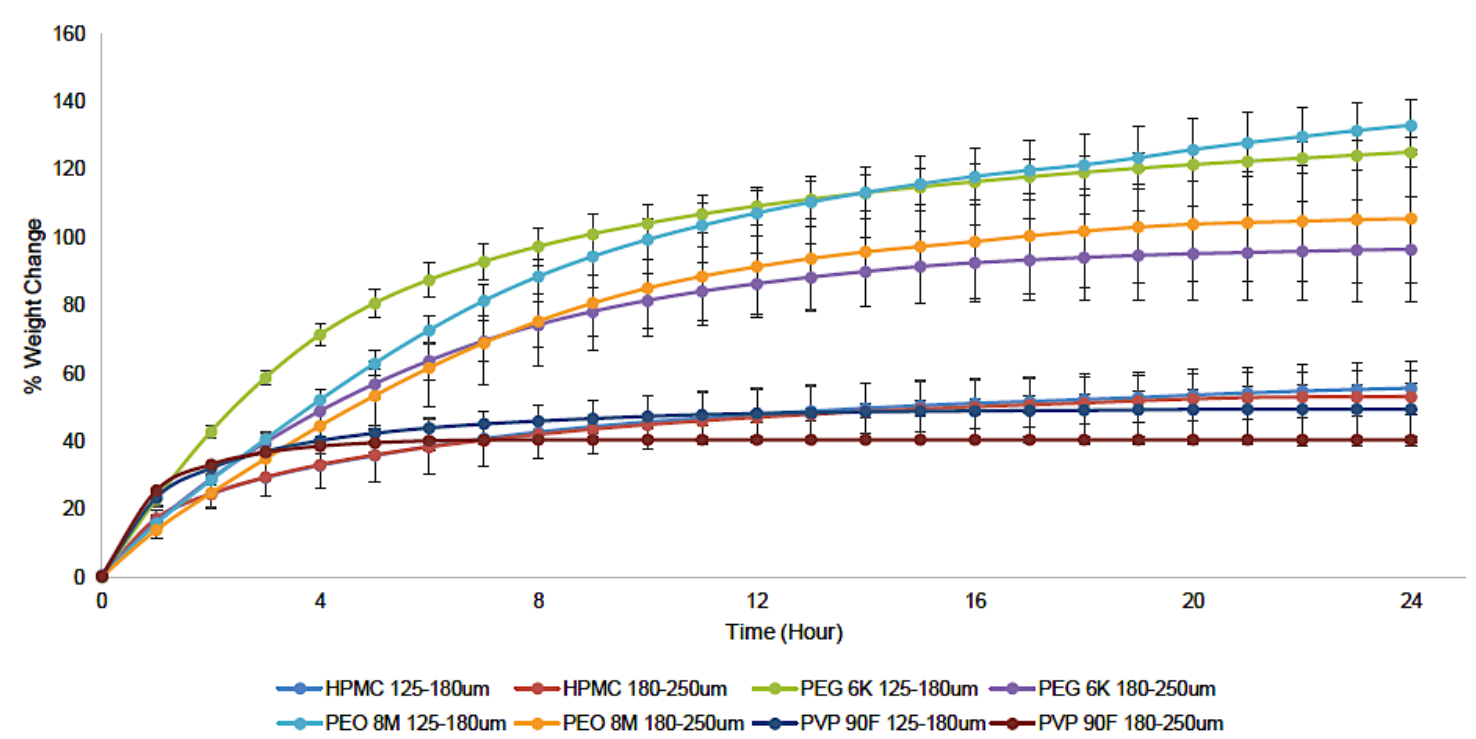

Figure 2 Percentage of weight changes from moisture uptake of mini-tablets.

HPMC and PVP 90F formulations displayed similar weight changes between the 2 size fractions, with weight increments between 40 and $50 \%$. The weight drastically increased within the first $\mathrm{h}$ and plateaued after $3 \mathrm{~h}$. PEG $6 \mathrm{~K}$ and PEO 8M formulations showed gradual weight changes with huge weight increments, where smaller sieve fraction mini-tablets absorbed more moisture than their counterparts. The smaller sieve fractions absorbed approximately $130 \%$ moisture, while the larger sieve fraction absorbed roughly $100 \%$ moisture compared to their initial weights. The larger sieve fraction approached a plateau at the end of the $24 \mathrm{~h}$ experiment, while the smaller sieve fraction continued to acquire moisture until the point of experiment termination.

\section{Drug release studies}

A modified flow-through dissolution apparatus was used for the dissolution study. This setup was based on a study by Brown et al. [12] on dissolution methodology for granules. Since the size of the minitablets and granules were roughly similar, this method was applicable for the mini-tablets. The minitablets were inserted into a $200 \mu \mathrm{L}$ chamber and flowed with STF at a rate of $50 \mu \mathrm{L} / \mathrm{min}$. The samples were collected hourly for $9 \mathrm{~h}$ and spectrophotometrically quantified at $278 \mathrm{~nm}$. Figure 2 shows the release profiles of all mini-tablets.

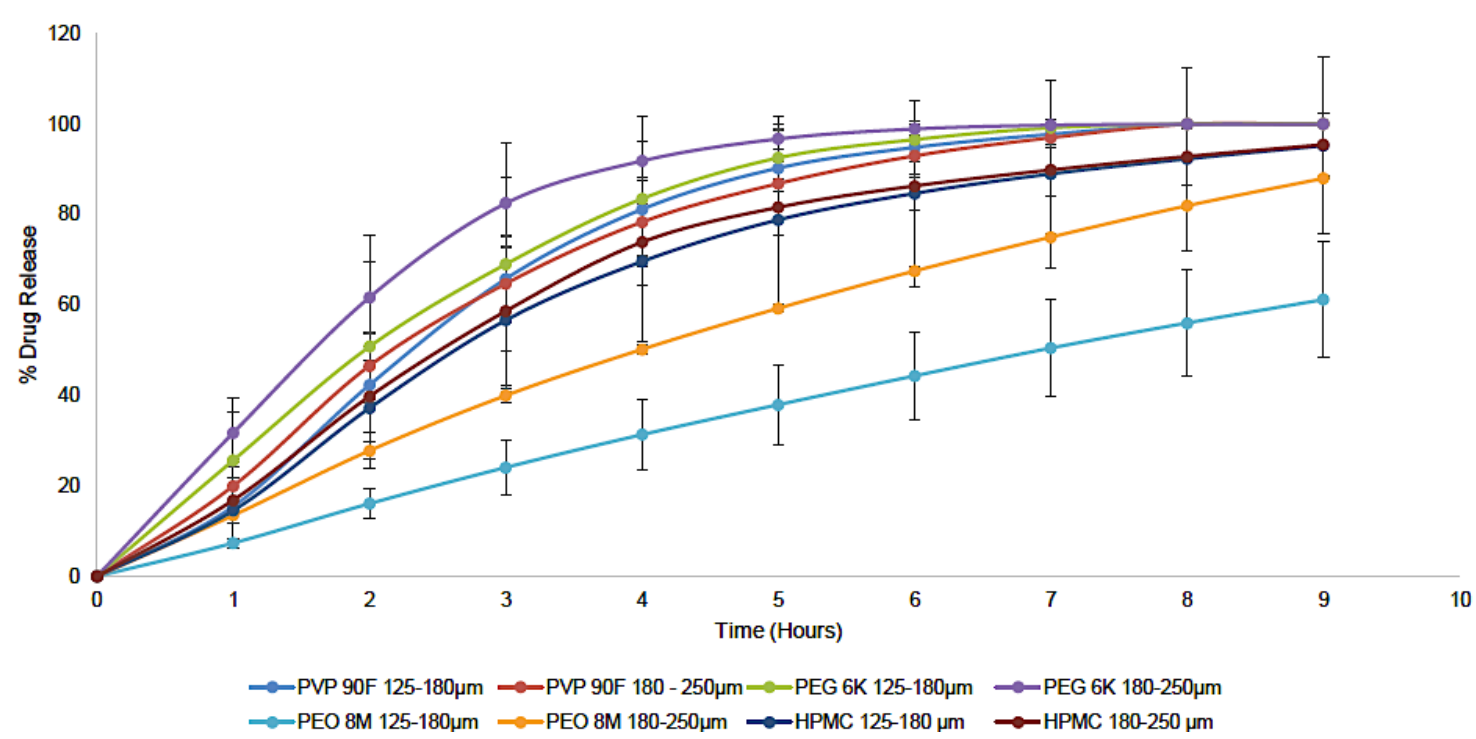

Figure 3 Drug release profiles of mini-tablets (mean $\pm \mathrm{SD}, \mathrm{n}=3$ ). 
All tablets were completely dissolved at the end of the dissolution experiment. Chloramphenicol from PVP 90F and PEG 6K mini-tablets of both sieve fractions was completely released. More than $80 \%$ of the chloramphenicol in HPMC mini-tablets (both sieve fractions) and PEO 8M (180 - $250 \mu \mathrm{m}$ sieve fraction) mini-tablets were released within $9 \mathrm{~h}$ timepoint. Only $60 \%$ of the chloramphenicol in PEO 8M (125 - $180 \mu \mathrm{m}$ sieve fraction) mini-tablets was released at the end of the experiment. PEO $8 \mathrm{M}$ formulation of both sieve fractions had almost linear release profiles compared to the rest of the formulations in the graph.

\section{Conclusions}

This study investigated the effect of particle size on the physical behavior and drug release characteristics of mini-tablets. All formulations produced high-quality mini-tablets, except for PVP 90F, which displayed a characteristic of high friability. PEG 6K and PEO 8M mini-tablets of the larger sieve fraction variation absorbed less moisture with faster release of drugs than their counterparts. Meanwhile, HPMC and PVP 90F mini-tablets of both sieve fractions showed similar pattern in term of moisture uptake and drug release profiles. PEO $8 \mathrm{M}$ mini-tablets of both sieve fractions displayed almost linear release profiles with the highest crushing strength than the rest of the formulations. In conclusion, the polymer's particle size in mini-tablets had a little bearing on the products' physical characteristics, although it could significantly affect the product's dissolution profile.

\section{Acknowledgements}

This work was supported by the Research Initiative Grant Scheme, International Islamic University Malaysia (RIGS 16-288-0452), Malaysia.

\section{References}

[1] P Lennartz and JB Mielck. Minitabletting: Improving the compactability of paracetamol powder mixtures. Int. J. Pharm. 1998; 173, 75-85.

[2] SM Majeed, MK Al-Shaheen, RN Al-Zidan and SM Mahmood. Multiple Unite Pellet Systems (MUPS) as drug delivery model. J. Drug Deliv. Ther. 2020; 10, 231-5.

[3] H Kotlowska, J Krotka, M Szymanska, B Kubiak, M Sznitowska and BN Nalluri. the use of novel tools for the assessment of powders and granules flow properties and for the analysis of minitablets compression process. Drug Dev. Ind. Pharm. 2020; 46, 547-56.

[4] C Tissen, K Woertz, J Breitkreutz and P Kleinebudde. Development of mini-tablets with $1 \mathrm{~mm}$ and 2 mm diameter. Int. J. Pharm. 2011; 416, 164-70.

[5] BP Commission. British pharmacopoeia 2020. The Stationery Office, London, 2019.

[6] AA El-Gawad, OA Soliman, SA Barker and GNS Girgis. Formulation and Evaluation of gel forming ocular minitablets containing piroxicam. Br. J. Pharm. Res. 2012; 2, 141-67.

[7] MRC Marques, R Loebenberg and M Almukainzi. Simulated biological fluids with possible application in dissolution testing. Dissolution Tech. 2011; 18, 15-28.

[8] W Weyenberg, A Vermeire, JP Remon and A Ludwig. Characterization and in vivo evaluation of ocular bioadhesive minitablets compressed at different forces. J. Contr. Release 2003; 89, 329-40.

[9] W Weyenberg, A Vermeire, J Vandervoort, JP Remon and A Ludwig. Effects of roller compaction settings on the preparation of bioadhesive granules and ocular minitablets. Eur. J. Pharm. Biopharm. 2005 ; 59, 527-36.

[10] M Sacchetti, R Teerakapibal, K Kim and EJ Elder. Role of water sorption in tablet crushing strength, disintegration, and dissolution. AAPS Pharm. Sci. Tech. 2017; 18, 2214-26.

[11] M Aulton and KMG Taylor. Aulton's Pharmaceutics: The design and manufacture of medicines. $5^{\text {th }}$ ed. Churchill Livingstone, Edinburgh, Scotland, 2017.

[12] CK Brown, HD Friedel, AR Barker, LF Buhse, S Keitel, TL Cecil, J Kraemer, JM Morris, C Reppas, MP Stickelmeyer C Yomota and VP Shah. Meeting report: FIP/AAPS joint workshop report: Dissolution/in vitro release testing of novel/special dosage forms. Dissolution Tech. 2011; 73, 338-53. 